\title{
The recombinant EGFR/CD13 bi-targeted fusion protein induces apoptosis and blocks tube formation
}

\author{
WEIJIN SHENG, JIANHUA GONG, MIN JIANG and YONGSU ZHEN \\ Institute of Medicinal Biotechnology, Chinese Academy of Medical Sciences \\ and Peking Union Medical College, Beijing 100050, P.R. China
}

Received April 18, 2017; Accepted October 9, 2017

DOI: $10.3892 /$ or.2017.6053

\begin{abstract}
Previously it was shown that the recombinant EGFR/CD13 bi-targeted fusion protein ER(Fv)-LDP-NGR which consists of an anti-EGFR scFv antibody fragment, a tri-cyclic NGR peptide, and a lidamycin-derived apoprotein, inhibited the proliferation of cancer cells and markedly suppressed tumor growth of breast carcinoma MCF-7 xenografts in athymic mice. This study investigated the mechanism of action of the fusion protein. Human breast cancer MCF-7 cells, lung adenocarcinoma A549 cells, and microvascular endothelial HMEC-1 cells were used for a series of assays and determinations. ER(Fv)-LDP-NGR downregulated the transcription and expression of the target proteins EGFR and CD13, and interfered with the intracellular EGFR signaling pathway, cell cycle signaling pathway and apoptotic pathway. It induced apoptosis, inhibited proliferation, caused cell cycle G2/M phase arrest, and suppressed cell migration. Accompanied by weakening the capability to degrade extracellular matrix, ER(Fv)-LDP-NGR depressed the invasion capacity of cancer cells. In addition, ER(Fv)-LDP-NGR prevented microvascular endothelial cells from tube formation, which is closely related to angiogenesis. In conclusion, the EGFR/CD13 bi-targeted fusion protein ER(Fv)-LDP-NGR displays multi-functional characteristics, acting on both cancer cells and endothelial cells. It might be an effective agent for targeted cancer therapy.
\end{abstract}

\section{Introduction}

Epidermal growth factor receptor (EGFR), a tyrosine kinase receptor of ErbB family, overexpressing on the plasma

Correspondence to: Professor Yongsu Zhen, Institute of Medicinal Biotechnology, Chinese Academy of Medical Sciences and Peking Union Medical College, 1 Tiantanxili, Beijing 100050, P.R. China

E-mail: zhenys@public.bta.net.cn

Abbreviations: scFv, single-chain variable fragment; NGR, a tripeptide consisting of asparagine, glycine and arginine; EGFR, epidermal growth factor receptor; CD13, aminopeptidase $\mathrm{N}$

Key words: EGFR, CD13, bi-targeted, multi-functional, antitumor membrane of most human malignant tumor cells, including those of the lung, head and neck, and pancreas, is regarded as a promising and validated molecular target in cancer drug therapy $(1,2)$. A serial of anti-EGFR agents including small-molecule kinase inhibitors and monoclonal antibodies have been applied for preclinical or clinical therapeutics for carcinomas in the form of drug combination $(3,4)$. For example, $43.7 \%$ of patients with non-small cell lung cancers treated with gefitinib as first-line therapy and platinum-based chemotherapy as second-line therapy were documented stable disease in NEJ002 (5). The combined application of erlotinib and bevacizumab remarkably promoted the proportion of 16-week progression-free survival (PFS16), the median PFS event and the median overall survival in advanced hepatocellular carcinomas (6). Afatinib-cetuximab demonstrated excellent clinical efficacy and manageable safety profile in heavily pretreated patients in advanced EGFR-mutant lung cancer with acquired resistance to erlotinib/gefitinib (7).

CD13 (aminopeptidase N, APN) is a glycoprotein that has been observed on the plasma membrane of vascular endothelial cells and tumor cells (8-10). CD13 boosts invasion of tumor cells through degradation of extracellular matrix and promotion of angiogenesis by promoting vascular endothelial cell invasion and adjusting related growth factors and cytokines $(11,12)$. Due to its special role in tumor progression, CD13 is an attractive target for the modulation of tumor microenvironment. Therefore, integration of EGFR and CD13 in cancer targeted therapy, the EGFR/CD13 bi-targeting strategy, might be of interest, as it acts on both tumor cells and tumor microenvironment. When EGFR and CD13 were considered together in the design of a recombinant fusion protein, the bi-targeting strategy can be realized by exerting potent antitumor efficacy through multiple biological modulations in cancer cells and surrounding microenvironment simultaneously with a single agent.

In our previous study, an EGFR/CD13 bi-targeted fusion protein ER(Fv)-LDP-NGR composed of a single chain Fv (scFv) targeting EGFR, a tri-cyclic NGR motif against CD13 and an apoprotein LDP serving as 'scaffold' had been prepared by DNA recombination. ER(Fv)-LDP-NGR at the dose of $10 \mathrm{mg} / \mathrm{kg}$ markedly inhibited the growth of MCF-7 xenografts in athymic mice (13). In addition, the enediyne-energized analogue of this fusion protein showed highly potent cytotoxicity to cancer cells. However, in the previous study, the efficacy of the non-enediyne-energized fusion protein ER(Fv)-LDP-NGR 
was not evaluated. The present study was set to investigate the mechanism of action of the EGFR/CD13 bi-targeted fusion protein ER(Fv)-LDP-NGR, including its effect on signaling pathways, induction of apoptosis and cell cycle arrest, inhibition of migration and invasion, and suppression of tube formation. Accordingly, the effects of ER(Fv)-LDP-NGR on tumor progression were evaluated and discussed.

\section{Materials and methods}

Cell culture. Human breast cancer MCF-7 cells and lung carcinoma A549 cells were cultured in RPMI-1640-Glutamax-I medium (Invitrogen Life Technologies, Carlsbad, CA, USA), containing $10 \%$ fetal bovine serum (Gibco/BRL, New York, $\mathrm{NY}$, USA) and penicillin and streptomycin at $100 \mathrm{U} / \mathrm{ml}$ and $100 \mu \mathrm{g} / \mathrm{ml}$ respectively. Human microvascular endothelial cell HMEC-1 was grown in DMEM-Glutamax-I medium (Invitrogen Life Technologies, Carlsbad, CA, USA), including $20 \%$ fetal bovine serum, $100 \mathrm{U} / \mathrm{ml}$ of penicillin and $100 \mu \mathrm{g} / \mathrm{ml}$ streptomycin.

Protein extraction and western blot analysis. The expression of target proteins and the influence of ER(Fv)-LDP-NGR on cell signaling pathway including EGFR signaling pathway, cell cycle pathway and apoptotic pathway were analyzed by western blotting. Tumor cells were lysed in cell lysis buffer (Beyotime Institute of Biotechnology, Haimen, China) supplemented with $1 \mathrm{mM}$ phenylmethylsulfonyl fluoride. Cellular proteins was clarified by high-speed centrifugation at $4^{\circ} \mathrm{C}$, and quantified with BCA protein assay kit (Thermo Fisher Scientific, Bremen, Germany). For each sample, $50 \mu \mathrm{g}$ of protein were applied in SDS-polyacrylamide gel electrophoresis (SDS-PAGE) and electrophoretically transferred onto polyvinylidene difluoride (PVDF) membranes (Merck Millipore, Belford, MA, USA). After being blocked with TBST (5\% skim milk, $20 \mathrm{mM}$ Tris- $\mathrm{HCl}, 150 \mathrm{mM} \mathrm{NaCl}$ and $0.1 \%$ Tween-20), the membranes were incubated with the primary antibodies (Cell Signaling Technology, Boston, MA, USA), and then with the HRP-conjugated secondary antibodies (Zhongshan Golden Bridge Biotechnology, Beijing, China). The specific bands were visualized with Immobilon Western Chemiluminescent HRP Substrate (Merck Millipore).

Cell proliferation assay. MTT assay was employed to examine proliferation activity of tumor cells. MCF-7, A549 and HMEC-1 cells were plated on 96 -well plates at a density of 5,000 cells/well and placed at $37^{\circ} \mathrm{C}$ in $5 \% \mathrm{CO}_{2}$ overnight for adhesion, then were incubated with different concentrations of fusion protein ER(Fv)-LDP-NGR for another $48 \mathrm{~h}$. After incubation with $20 \mu \mathrm{l}$ of MTT $(5 \mathrm{mg} / \mathrm{ml})$ for $4 \mathrm{~h}$, the culture supernatant was removed, and $150 \mu \mathrm{l}$ of DMSO was added into the wells. The cell plates were read at $570 \mathrm{~nm}$ with a microplate reader (Thermo Fisher Scientific), then the survival rate and the $50 \%$ inhibitory concentration $\left(\mathrm{IC}_{50}\right)$ was calculated.

Determination of EGFR and CD13 mRNA levels. Reverse transcription-polymerase chain reaction (RT-PCR) was applied to assess mRNA levels of targeted protein in tumor cells. MCF-7, A549 and HMEC-1 cells were incubated in complete medium with 0.1 or $1 \mu \mathrm{M}$ ER(Fv)-LDP-NGR for
48 h. Total RNA of tumor cells was isolated with TRIzol reagent (Takara Bio Inc, Dalian, China), and mRNA was purified with PolyATract mRNA Isolation system (Promega Corp., Madison, WI, USA). cDNA was synthesized by reverse transcription and EGFR, $C D 13$ and $\beta$-actin gene fragments were further amplified by polymerase chain reaction using the primers EGFR (sense, 5'-TCCCCGTAATTATGTGGTGAC AGATC-3'; antisense, 5'-ACCCCTAAATGCCACCGGC-3'), CD13 (sense, 5'-CCTTCAACCTGGCCAGTGC-3'; antisense, 5'-CGTCTTCTCCAGGGCTTGCTCCAG-3') and $\beta$-actin (sense, 5'-CTGGGACGACATGGAGAAAA-3'; antisense, 5'-AAGGAAGGCTGGAAGAGTGC-3') (Invitrogen Life Technologies, Shanghai, China), respectively. The amplified DNA fragments were separated in $1 \%$ agarose gel with GoodView nucleic acid stain (SBS Genetech Corp., Beijing, China) and visualized under UV.

Cell cycle analysis. Cell cycle of MCF-7 and A549 cells treated with ER(Fv)-LDP-NGR was detected by flow cytometry (Becton-Dickinson and Co., Franklin Lakes, NJ, USA). The cells treated with 0.1 or $1 \mu \mathrm{M}$ ER(Fv)-LDP-NGR for $48 \mathrm{~h}$ were collected and fixed with $70 \%$ ethanol, and then resuspended in dye containing $100 \mu \mathrm{g} / \mathrm{ml}$ of RNaseA and $25 \mu \mathrm{g} / \mathrm{ml}$ of propidium iodide (PI). Cell cycle analysis was performed following a light proof dyeing at room temperature.

Apoptosis analysis. The effect of fusion protein on apoptosis of tumor cells was examined by Hoechst 33385 staining and Annexin V-FITC/PI analysis, respectively. MCF-7 and A549 cells seeded on coverslips were incubated with $1 \mu \mathrm{M}$ ER(Fv)-LDP-NGR for 48 h. Fixed with $4 \%$ paraformaldehyde at room temperature, the cells were dyed with Hoechst 33385 (Sigma-Aldrich, Santa Clara, CA, USA) for $10 \mathrm{~min}$, then were observed under a fluorescence microscope with x200 magnification and recorded by a camera (Nikon Corp., Tokyo, Japan).

For Annexin V-FITC/PI analysis, tumor cells with ER(Fv)LDP-NGR treatment were collected at 50,000 cells/tube density. After washing three times with cold PBS, the cell pellets were resuspended with binding buffer, followed by staining with Annexin V-FITC/PI (Biosea Biotechnology, Beijing, China). The fluorescence was determined by flow cytometry in an hour.

In vitro cell migration and cell invasion assay. Transwell permeable supports (Corning Inc., Corning, NY, USA) with polyester filter membrane were used to assess migration and invasion capacity of tumor cells. The Transwell compartments, 24-well format, with $8 \mu \mathrm{m}$ pore size insert, were incubated with RPMI-1640 medium for an initial equilibrium. RPMI-1640 $(0.1 \mathrm{ml})$ with $2 \% \mathrm{FBS}$ containing $1 \times 10^{5}$ tumor cells and $0.6 \mathrm{ml}$ of RPMI-1640 with $20 \%$ FBS were added into the wells and the Transwell inserts, respectively. After ER(Fv)-LDP-NGR was added, the cells were incubated in the Transwell plates at $37^{\circ} \mathrm{C}, 5 \% \mathrm{CO}_{2}$ for $24 \mathrm{~h}$, which enabled the cells to migrate toward the underside of the insert filters. The inserts were carefully taken out and cells were fixed with methanol for 3 min and stained with $0.1 \%$ crystal violet for $30 \mathrm{~min}$. Cells remaining on the upper side of filter membrane were gently removed with a cotton swab after washing the insert with PBS, then the inserts were 


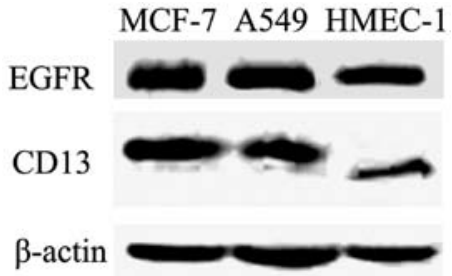

Figure 1. Expression of EGFR and CD13 in MCF-7, A549 and HMEC-1 cells.

observed under a microscope and recorded by a camera. The membrane was cut and immersed into $33 \%$ acetic acid to dissolve crystal violet. The absorbance at $570 \mathrm{~nm}$ was read with a microplate reader, and the relative migration of tumor cells was calculated.

Transwell permeable supports were coated with pre-cooled Matrigel (Becton-Dickinson and Co.) before cell invasion assay. Matrigel melted at $4^{\circ} \mathrm{C}$ was added into the Transwell inserts and incubated at $37^{\circ} \mathrm{C}$ for polymerization on filter membrane. The subsequent experimental operation manipulation and calculation of the relative invasion were carried out as cell migration assay.

Tube formation assay. Tube formation is a fast and easy assay to examine the angiogenic/anti-angiogenic properties of drugs, which can be conveniently performed in any cell culture lab. Pre-cooled Matrigel was transferred to a 48-well plate and incubated at $37^{\circ} \mathrm{C}$ for solidification. HMEC- 1 cells $\left(4 \times 10^{5}\right)$ were counted and suspended in $200 \mu \mathrm{l}$ culture media with fusion protein, then were transferred to the 48 -well plate. After incubation at $37^{\circ} \mathrm{C}, 5 \% \mathrm{CO}_{2}$ for $48 \mathrm{~h}$, the number of endothelial tubes and their size were observed under a microscope and photographed. Tube formation was quantified by measuring tubes per well using Image-Pro Plus software.

Statistical analysis. The data are presented as the mean \pm standard deviation (SD). Statistical comparisons between groups were analyzed by Student's t-test, and a significant difference was set at $\mathrm{P}<0.05$.

\section{Results}

Expression of EGFR and CD13 in tumor cells and microvascular endothelial cells. The expression of the target proteins EGFR and CD13 in MCF-7, A549 and HMEC-1 cells was analyzed by western blotting. Overexpression of EGFR and CD13 was observed in both tumor cells and microvascular endothelial cells, while CD13 isoform in HMEC-1 cells replaced CD13 in MCF-7 and A549 cells (Fig. 1).

Effect of the bispecific fusion protein on cell proliferation. The anti-proliferative activity of ER(Fv)-LDP-NGR was measured by MTT assay. As shown in Fig. 2, ER(Fv)-LDP-NGR at micromolar concentration could inhibit the proliferation of tumor cells and microvascular endothelial cells with $\mathrm{IC}_{50}$ values of $10^{-7}-10^{-6} \mathrm{M}$.

Downregulation of the $M R N A$ and protein levels of EGFR and $C D 13$. The regulation of EGFR and CD13 gene transcription

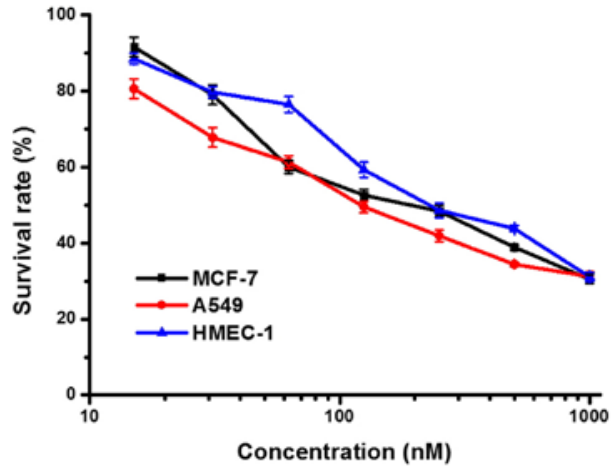

Figure 2. Effect of the bispecific fusion protein on cell proliferation. MCF-7, A549 and HMEC-1 cells were treated with different concentrations of $\mathrm{ER}(\mathrm{Fv})-\mathrm{LDP}-\mathrm{NGR}$ for $48 \mathrm{~h}$. The inhibitory rate was evaluated by MTT assay.

and expression was assessed by RT-PCR and western blotting, respectively. As Fig. 3 shows, ER(Fv)-LDP-NGR caused a dose-dependent decrease in mRNA and protein levels in both tumor cells and microvascular endothelial cells, while no obvious change of $\beta$-actin was observed.

Regulation of signal transduction pathways. The regulations of ER(Fv)-LDP-NGR on EGFR, cell cycle and apoptosis signaling pathways in tumor cells were analyzed by western blotting (Fig. 4). For EGFR-positive MCF-7 cells, ER(Fv)-LDP-NGR dose-dependently downregulated the expression level of EGFR and its phosphorylation. In addition, the phosphorylation of corresponding protein kinases, including Akt, Raf, ERK, MAPK, was accordingly reduced, except SAPK/JNK, which could be induced by cellular stress response.

ER(Fv)-LDP-NGR upregulated the phosphorylation of ATM/ ATR kinase, which was followed by activation of checkpoint kinases (Chk1 and Chk2) and p53, and then downregulation of $\mathrm{Cdc} 2 / \mathrm{Cyclin} \mathrm{B} 1$ complexes. No obvious decrease was observed in $\mathrm{CDK} 2 / \mathrm{Cyclin} \mathrm{A}$, which are associated with $\mathrm{S}$ phase.

As shown in Fig. 4C, a slight increased apoptosis induction of ER(Fv)-LDP-NGR resulted from the reverse effects of intrinsic apoptotic pathway and extrinsic apoptotic pathway. Although ER(Fv)-LDP-NGR inhibited the extrinsic pathway that presented as decrease of caspase-10, it activated the intrinsic pathway characterized by the permeabilisation of the mitochondria and release of cytochrome $c$ into the cytoplasm. Cellular stress response and DNA damage activated proapoptotic protein Bax, which subsequently resulted in the activation of a series of cysteinyl aspartate specific proteinases (the initiator caspase-9 and the executioner protein caspase-3, and -7) and PARP cleavage. The hydrolysis of protein finally led to DNA fragmentation, cell shrinkage, and membrane bleb formation.

Effect of the bispecific fusion protein on cell cycle progression. The cell cycle distribution of tumor cells treated with ER(Fv)-LDP-NGR was detected by measuring PI fluorescence with flow cytometry. ER(Fv)-LDP-NGR caused G2/M phase arrest of tumor cells, and the extent of arrest was concentration-dependent. Treated with $1 \mu \mathrm{M}$ ER(Fv)-LDP-NGR, 82\% of MCF-7 cells and $65.2 \%$ of A549 cells were, respectively 
A $\mathrm{MCF}-7$

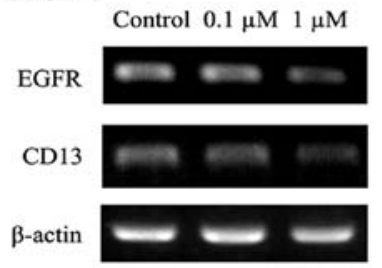

B MCF-7

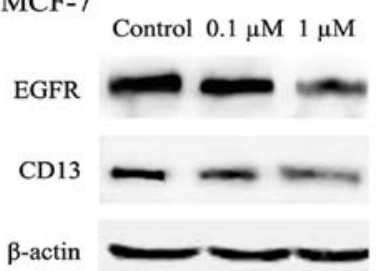

A549

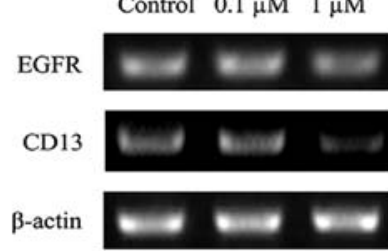

A549

Control $\quad 0.1 \mu \mathrm{M} \quad 1 \mu \mathrm{M}$

EGFR

CD13

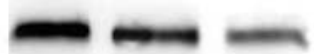

$\beta$-actin
HMEC-1

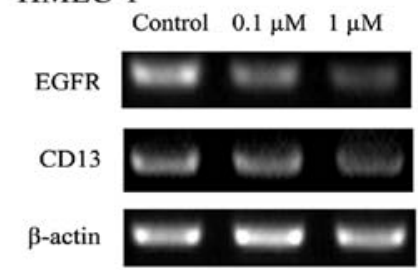

HMEC-1

Control $0.1 \mu \mathrm{M} 1 \mu \mathrm{M}$

EGFR

CD13

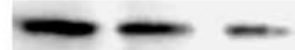

$\beta$-actin

Figure 3. Effect of the bispecific fusion protein on mRNA and protein levels of EGFR and CD13. MCF-7 and A549 cells were treated with 0.1 or $1 \mu \mathrm{M}$ ER(Fv)-LDP-NGR for $48 \mathrm{~h}$. RT-PCR and western blotting were carried out to test the changes in mRNA (A) and protein (B) levels of target proteins.

A

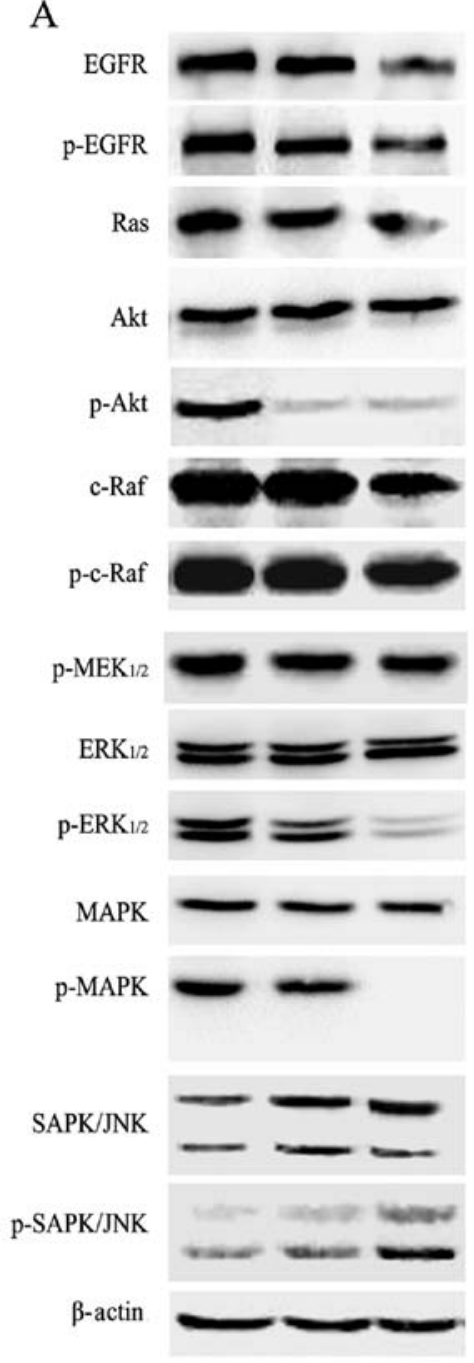

B

ATM

ATR

p-ATM

p-ATR

Chk1

Chk

Chk2

p-Chkl

p-Chk2

Cdc25C

p5

p-p53

Cdc2

Cdc2

p-Cdc2

Cyclin B1

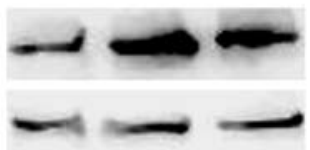

$\mathrm{C}$

Caspase-10
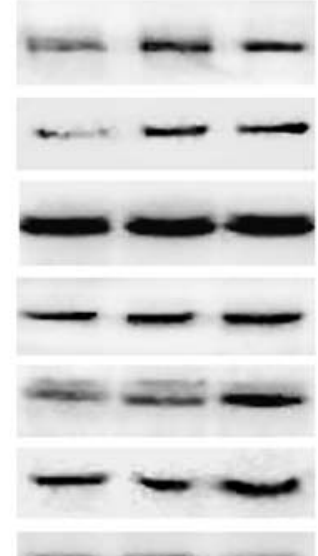

Caspase-3
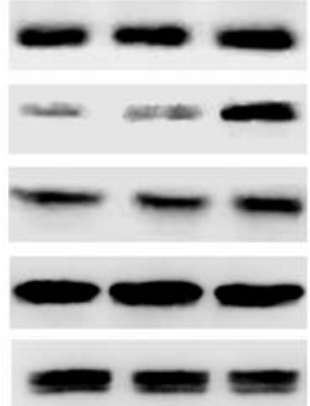

Caspase-9

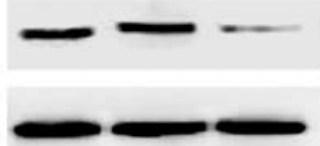

PARP
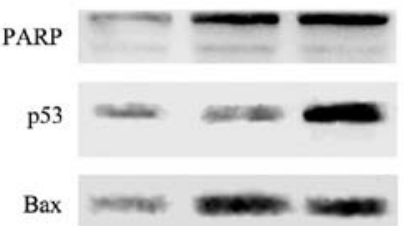

Bcl-2

NF- $\kappa \mathrm{B}$

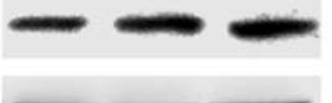

NF-x
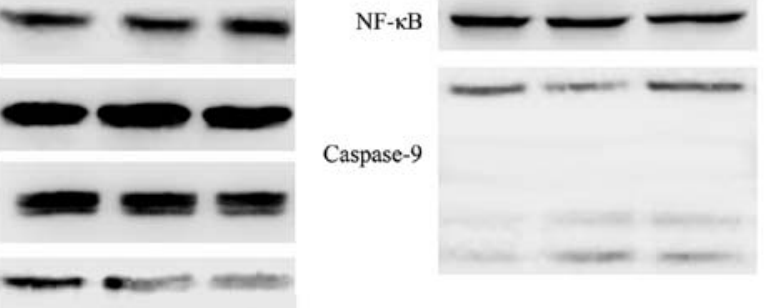

p2.

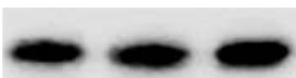

CDK2

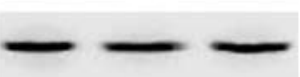

Cyclin A

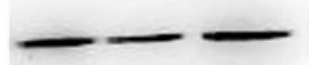

Figure 4. Effect of the bispecific fusion protein on signaling pathways of tumor cells. MCF-7 cells were incubated with 0.1 or $1 \mu \mathrm{M}$ ER(Fv)-LDP-NGR for $48 \mathrm{~h}$, and western blotting were performed to analyze the influence of the fusion protein on EGFR signaling pathway (A), cell cycle pathway (B) and apoptotic pathway (C). 
A

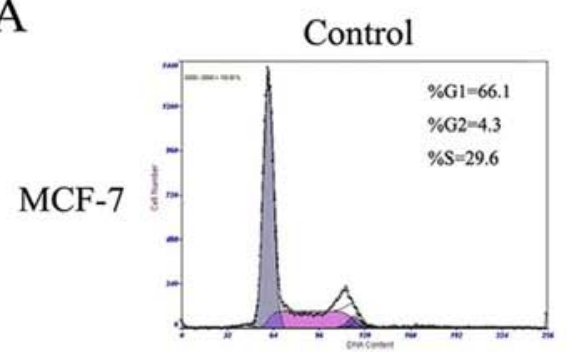

Control

A549

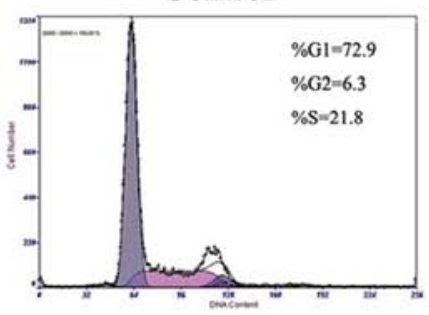

$0.1 \mu \mathrm{M}$

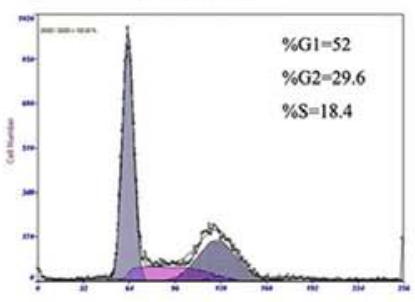

$0.1 \mu \mathrm{M}$

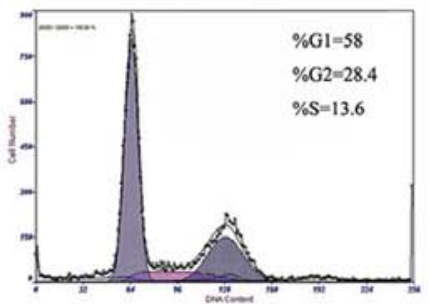

$1 \mu \mathrm{M}$

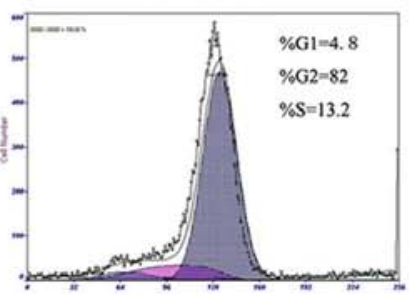

$1 \mu \mathrm{M}$

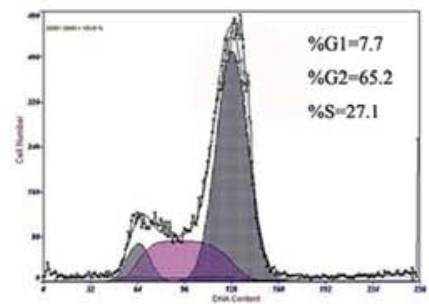

B

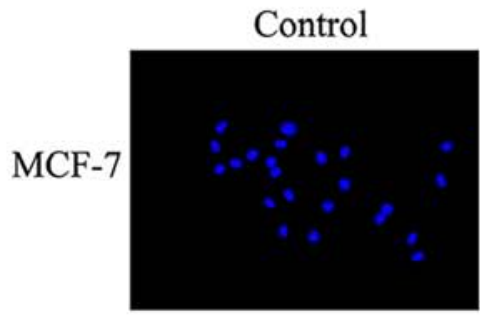

Control

A549

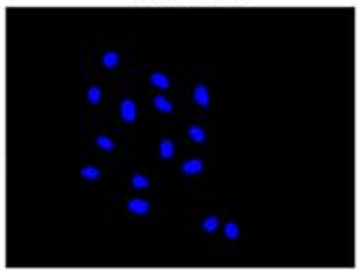

ER(Fv)-LDP-NGR

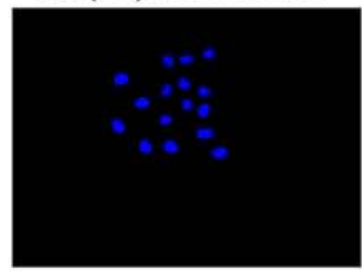

ER(Fv)-LDP-NGR

C
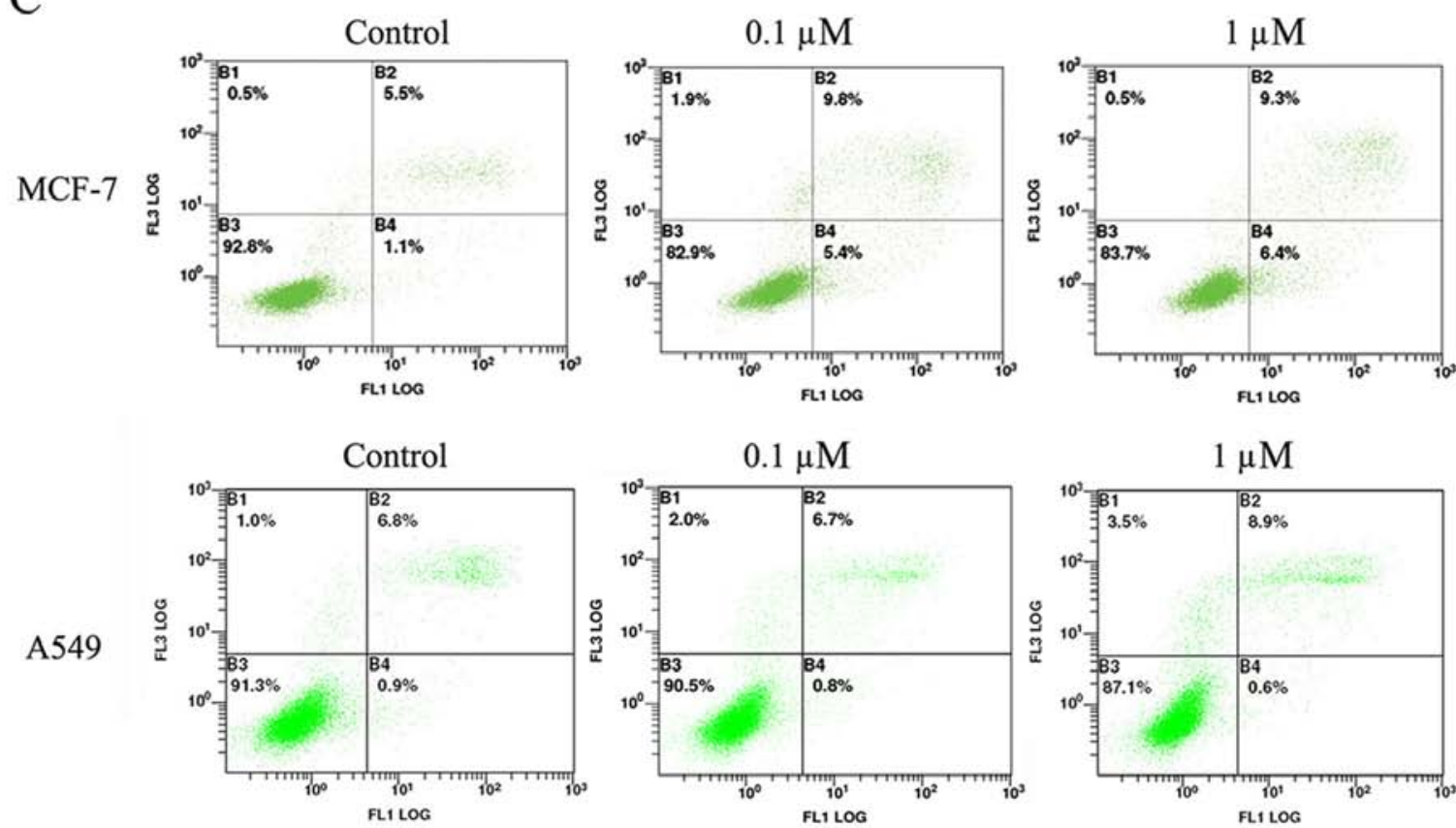

Figure 5. Effect of the bispecific fusion protein on cell cycle distribution and apoptosis of tumor cells. MCF-7 and A549 cells were treated with 0.1 or $1 \mu \mathrm{M}$ ER(Fv)-LDP-NGR for $48 \mathrm{~h}$. Cell cycle distribution was measured by flow cytometry after PI staining, and effects on cell apoptosis were measured by Hoechst staining and FITC-Annexin V/PI apoptosis assay. (A) Cell cycle distribution of MCF-7 and A549 cells. (B) Representative images of MCF-7 and A549 cells after treatment. (C) Phase diagrams of MCF-7 and A549 cells after FITC-Annexin V/PI staining. 
A

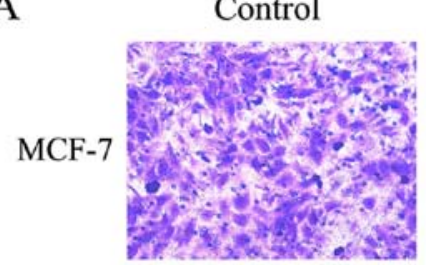

Control

A549

$\mathrm{C}$

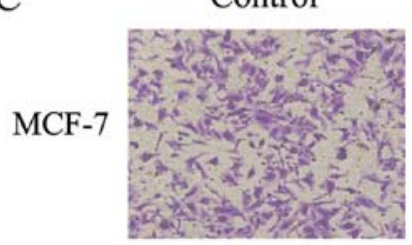

Control

A549

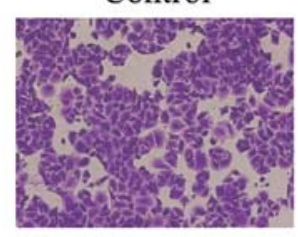

ER(Fv)-LDP-NGR

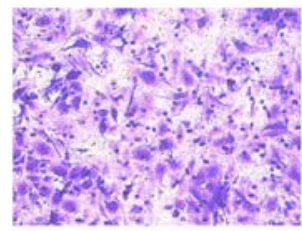

ER(Fv)-LDP-NGR

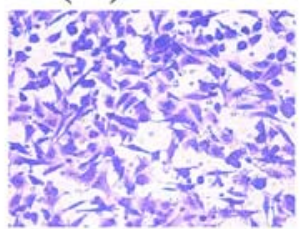

ER(Fv)-LDP-NGR

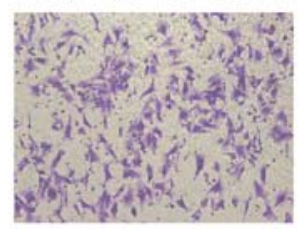

ER(Fv)-LDP-NGR

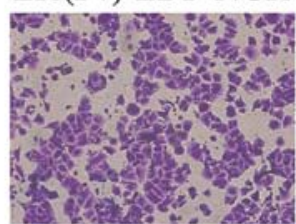

B

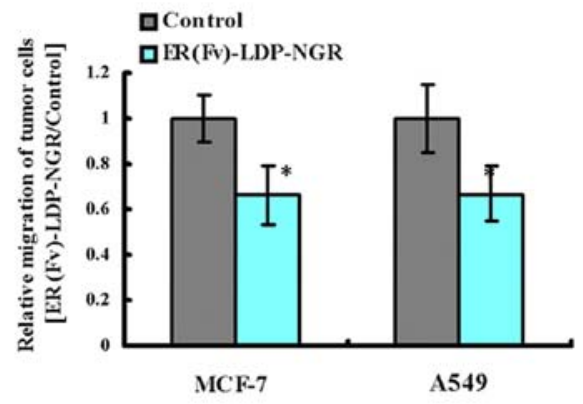

D

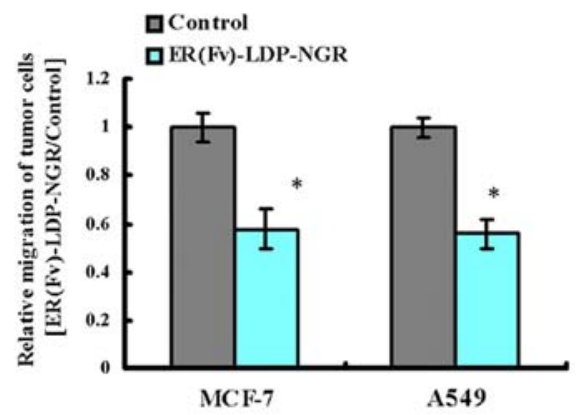

Figure 6. Effect of the bispecific fusion protein on the migration and invasion of tumor cells. MCF-7 and A549 cells were treated with $1 \mu$ MER(Fv)-LDP-NGR for $24 \mathrm{~h}$, and Transwell permeable supports with polyester filter membrane were used to assess migration and invasion capacity of tumor cells. (A and C) Microscopic images of tumor cells stained with crystal violet in migration and invasion assay. (B and D) The relative migration and invasion of tumor cells treated with ER(Fv)-LDP-NGR and the negative controls ( $\mathrm{p}<0.05$ vs. the controls). Data are shown as the mean \pm SD from three independent experiments.

A

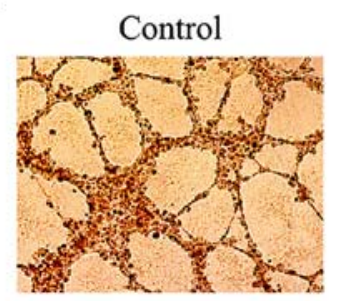

ER(Fv)-LDP-NGR

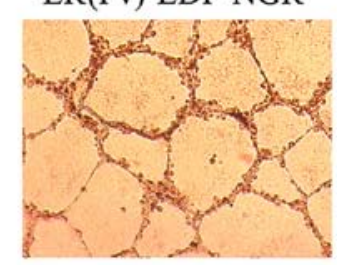

B

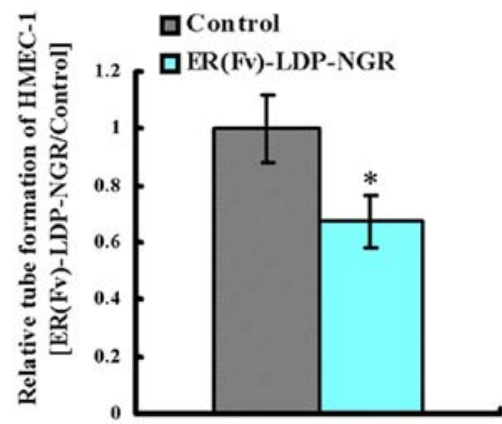

Figure 7. Effect of the bispecific fusion protein on microvascular endothelial cells. (A) Microscopic images of tube formation by HMEC-1 cells after $1 \mu \mathrm{M}$ ER(Fv)-LDP-NGR treatment. (B) The relative tube formation of HMEC-1 cells treated with ER(Fv)-LDP-NGR and the negative controls ("p $<0.05$ vs. the controls). Data are shown as the mean \pm SD from three independent experiments.

blocked at G2 phase, and a decrease of proportion MCF-7 cells in $\mathrm{S}$ phase was observed (Fig. 5A).

Induction of apoptosis by the bispecific fusion protein. As the result of Hoechst 33385 (Fig. 5B) and Annexin V-FITC/PI staining (Fig. 5C), higher concentrations of ER(Fv)-LDP-NGR induced early and late apoptosis in tumor cells. Compared with the control, a part of MCF-7 and A549 cells treated with $1 \mu \mathrm{M}$ ER(Fv)-LDP-NGR showed enlarged cells with a single giant nucleus and condensed nuclei.
Inhibition of migration and invasion by the bispecific fusion protein. Transwell assay was applied to assess the migration and invasion capacity of tumor cells. ER(Fv)-LDP-NGR at $1 \mu \mathrm{M}$ inhibited tumor cells migrating from the upper surface of polyester filter membrane to the lower surface, with an inhibitory rate of $33.7 \%$ in MCF-7 cells and $32.8 \%$ in A549 cells, respectively (Fig. 6A and B). ER(Fv)-LDP-NGR also suppressed the invasion capacity of tumor cells, and reduced the number of cells passing through Matrigel. Only 58.0\% of MCF-7 cells and $56.3 \%$ of A549 cells incubated with 
ER(Fv)-LDP-NGR could reach the lower surface of filter membrane (Fig. 6C andD).

Effects of the bispecific fusion protein on microvascular endothelial cells. Tube formation assay, a classical angiogenesis assay in vitro, was employed to detect the anti-angiogenesis activity of the fusion protein ER(Fv)-LDP-NGR. As shown in Fig. 7, HMEC-1 cells normally aligned and formed tube-like structures, while a decrease in the number of tubes and branch points was observed following treatment with the fusion protein, indicating that ER(Fv)-LDP-NGR could suppress the angiogenic capacity of microvascular endothelial cells.

\section{Discussion}

The occurrence and development of tumors are the result of imbalances in both tumor cells and surrounding microenvironment, such as extracellular matrix, angiogenesis, vascular permeability and immunocytes. The integrated treatment targeting both cancer cells and tumor microenvironment has been considered a promising therapeutic strategy. Accordingly, bi-functional or multi-functional tyrosine kinase inhibitors, bispecific monoclonal antibodies, antibody-based conjugates and recombinant fusion proteins have been developed and reported as new antitumor drugs. Among the potential drug targets, EGFR and CD13 have attracted much attention for the reason that they can be combined with other targets to produce bi-targeted therapeutics. As reported, the bispecific diphtheria toxin-based recombinant cytotoxin (DTEGF13) targeting human EGFR and interleukin-13 selectively killed the human glioblastoma U87-luc cells and other glioblastoma cells in vitro, and two injections of DTEGF13 at a dose of $0.5 \mathrm{mg} / \mathrm{kg}$ resulted in eradication of the aggressive brain tumor in $50 \%$ of the rats that could survive with tumor-free status for $>110$ days after tumor inoculation (14). Compared with unsubstituted zinc(II) phthalocyanine, erlotinib-zinc(II) phthalocyanine conjugates could effectively target EGFR-overexpressing tumor cells, and showed significantly high photo-cytotoxicity toward HepG2 cells (15). A bispecific antibody (BsAb), consisting of anti-CD3 Fab' and anti-CD13 Fab', was shown to react with both CD3 T cells and $\mathrm{CD}_{13}{ }^{+}$acute myeloid leukemia (AML) cells. Cytokinestimulated peripheral blood mononuclear cells combining with $\mathrm{BsAb}$ exhibited enhanced cytotoxicity against $\mathrm{CD}^{+} 3^{+} \mathrm{AML}$ cells, suggesting that BsAb might be effective in the ex vivo purging of $\mathrm{CD}^{2} 3^{+} \mathrm{AML}$ cells in autologous bone marrow transplantation (16). A large number of studies on EGFR-based or CD13-based bi-targeted conjugates or fusion proteins have been documented; however, few of them are related to EGFR/CD13 bi-targeting simultaneously in a single agent.

$\mathrm{ER}(\mathrm{Fv})$-LDP-NGR is an EGFR/CD13 bi-targeting fusion protein with multiple antitumor activities. EGFR is regarded as one the most important targets on the plasma membrane of cancer cells and has been validated as a drug target for cancer therapeutics. CD13 expressed on the membrane of both tumor cells and vascular endothelial cells is associated with the invasion of tumor cells and tumor angiogenesis. This study showed that ER(Fv)-LDP-NGR reduced the phosphorylation of EGFR by blocking the binding of the receptor to its ligand and affected the hydrolysis capacity of CD13. The fusion protein not only decreased the mRNA and protein levels of EGFR and
CD13 but also weakened their biological activity, implying that ER(Fv)-LDP-NGR suppress the transcription and translation of target genes. The multiple antitumor capability of the fusion protein was embodied in anti-proliferation, invasion blocking and tube formation inhibition. For cancer cells, ER(Fv)-LDP-NGR could interfere with the intracellular EGFR signaling pathway and related pathways by downregulating the expression and phosphorylation of EGFR, resulting in proliferation inhibition, cell cycle arrest, apoptosis induction, and blockade of cell migration. Due to the decrease in CD13 expression, the capacity of the cells degrade extracellular matrix was weakened; consequently, cancer cell invasion was inhibited. In addition, the downregulation of CD13 by ER(Fv)-LDP-NGR could interfere with the formation of tube network structures of microvascular endothelial cells, which would lead to the suppression of angiogenesis.

The fusion protein ER(Fv)-LDP-NGR containing an anti-EGFR scFv and a tri-cyclic NGR peptide is endowed with the capability to aim at two targets, the EGFR and CD13. In the present study, the scFv against EGFR was obtained by multiround screening of the recombinant phage antibody library in our laboratory, and it displays specific binding activity to purified EGFR protein and EGFR on the membrane of carcinoma cells (17). The phage peptide library screening product CNGRC shows a greater affinity for CD13 than GNGRG and linear NGR with the same function (18), and improves therapeutic efficacy of immunotherapeutic and antiangiogenic molecules $(19,20)$. The scFv and CNGRC portions in the fusion protein blocks EGFR and CD13, respectively, and simultaneously exert antitumor efficacy by growth inhibition and microenvironment modulation. Additionally, the scFv and the NGR peptide constitute an efficient EGFR/CD13-targeted delivery carrier, which can transport a variety of potent 'warhead' drugs (such as lidamycin, maytansine, and auristatin) into solid tumors to realize targeted therapy of cancer (21-23).

In conclusion, the EGFR/CD13 bi-targeted fusion protein ER(Fv)-LDP-NGR can simultaneously affect tumor cells and tumor microenvironment through inhibition of cancer cell proliferation, induction of apoptosis, blockade of invasion and suppression of tube formation. In addition, ER(Fv)-LDP-NGR may be used as an EGFR/CD13 bi-targeted carrier for various 'warhead' agents to construct antibody-based and ligandbased conjugates with high therapeutic efficacy.

\section{Acknowledgements}

This study was supported by grants from 'Significant New Drug Development' Major Science and Technology Projects of China (nos. 2012ZX09301002-001-024-05 and 2013ZX09102064).

\section{References}

1. Levitzki A: EGF receptor as a therapeutic target. Lung Cancer 41: S9-S14, 2003.

2. Brand TM, Iida M, Li C and Wheeler DL: The nuclear epidermal growth factor receptor signaling network and its role in cancer. Discov Med 12: 419-432, 2011.

3. Fury MG, Xiao H, Sherman EJ, Baxi S, Smith-Marrone S, Schupak K, Gewanter R, Gelblum D, Haque S, Schoder H, et al: Phase II trial of bevacizumab + cetuximab + cisplatin with concurrent intensity-modulated radiation therapy for patients with stage III/IVB head and neck squamous cell carcinoma. Head Neck 38: E566-E570, 2016. 
4. Han JY, Lee SH, Lee GK, Yun T, Lee YJ, Hwang KH, Kim JY and Kim HT: Phase I/II study of gefitinib (Iressa(®)) and vorinostat (IVORI) in previously treated patients with advanced non-small cell lung cancer. Cancer Chemother Pharmacol 75: 475-483, 2015.

5. Miyauchi E, Inoue A, Kobayashi K, Maemondo M, Sugawara S, Oizumi S, Isobe H, Gemma A, Saijo Y, Yoshizawa $\mathrm{H}$, et al; North-East Japan Study Group: Efficacy of chemotherapy after first-line gefitinib therapy in EGFR mutation-positive advanced non-small cell lung cancer-data from a randomized Phase III study comparing gefitinib with carboplatin plus paclitaxel (NEJ002). Jpn J Clin Oncol 45: 670-676, 2015.

6. Thomas MB, Morris JS, Chadha R, Iwasaki M, Kaur H, Lin E, Kaseb A, Glover K, Davila M and Abbruzzese J: Phase II trial of the combination of bevacizumab and erlotinib in patients who have advanced hepatocellular carcinoma. J Clin Oncol 27: 843-850, 2009.

7. Janjigian YY, Smit EF, Groen HJ, Horn L, Gettinger S, Camidge DR, Riely GJ, Wang B, Fu Y, Chand VK, et al: Dual inhibition of EGFR with afatinib and cetuximab in kinase inhibitor-resistant EGFR-mutant lung cancer with and without T790M mutations. Cancer Discov 4: 1036-1045, 2014.

8. Tokuhara T, Hattori N, Ishida H, Hirai T, Higashiyama M, Kodama K and Miyake M: Clinical significance of aminopeptidase $\mathrm{N}$ in non-small cell lung cancer. Clin Cancer Res 12: 3971-3978, 2006.

9. Ranogajec I: Gelatinase (MMP-2, MMP-9) and Aminopeptidase N/CD13 in Breast Carcinoma. LAP Lambert Academic Publishing, 2014.

10. Nohara S, Kato K, Fujiwara D, Sakuragi N, Yanagihara K, Iwanuma Y and Kajiyama Y: Aminopeptidase N (APN/CD13) as a target molecule for scirrhous gastric cancer. Clin Res Hepatol Gastroenterol 40: 494-503, 2016.

11. Saiki I, Fujii H, Yoneda J, Abe F, Nakajima M, Tsuruo T and Azuma I: Role of aminopeptidase N (CD13) in tumor-cell invasion and extracellular matrix degradation. Int J Cancer 54: 137-143, 1993.

12. Fukasawa K, Fujii H, Saitoh Y, Koizumi K, Aozuka Y, Sekine K, Yamada M, Saiki I, Nishikawa K, et al: Aminopeptidase N (APN/CD13) is selectively expressed in vascular endothelial cells and plays multiple roles in angiogenesis. Cancer Lett 243: 135-143, 2006

13. Sheng W, Shang Y, Li L and Zhen Y: An EGFR/CD13 bispecific fusion protein and its enediyne-energized analog show potent antitumor activity. Anticancer Drugs 25: 82-91, 2014.
14. Oh S, Ohlfest JR, Todhunter DA, Vallera VD, Hall WA, Chen H and Vallera DA: Intracranial elimination of human glioblastoma brain tumors in nude rats using the bispecific ligand-directed toxin, DTEGF13 and convection enhanced delivery. J Neurooncol 95: 331-342, 2009.

15. Zhang FL, Huang Q, Liu JY, Huang MD and Xue JP: Moleculartarget-based anticancer photosensitizer: Synthesis and in vitro photodynamic activity of erlotinib-zinc(II) phthalocyanine conjugates. ChemMedChem 10: 312-320, 2015.

16. Kaneko T, Fusauchi Y, Kakui Y, Masuda M, Akahoshi M, Teramura M, Motoji T, Okumura K, Mizoguchi H and Oshimi K: A bispecific antibody enhances cytokine-induced killer-mediated cytolysis of autologous acute myeloid leukemia cells. Blood 81: 1333-1341, 1993.

17. Sheng WJ, Shang BY, Miao QF and Zhen YS: Construction of a single-chain Fv antibody against epidermal factor receptor and its antitumor activity. Chung Kuo Yao Hsueh Tsa Chih 46: 1393-1398, 2011 (In Chinese).

18. Colombo G, Curnis F, De Mori GM, Gasparri A, Longoni C, Sacchi A, Longhi R and Corti A: Structure-activity relationships of linear and cyclic peptides containing the NGR tumor-homing motif. J Biol Chem 277: 47891-47897, 2002.

19. Curnis F, Sacchi A, Borgna L, Magni F, Gasparri A and Corti A: Enhancement of tumor necrosis factor alpha antitumor immunotherapeutic properties by targeted delivery to aminopeptidase N (CD13). Nat Biotechnol 18: 1185-1190, 2000.

20. Jiang W, Jin G, Ma D, Wang F, Fu T, Chen X, Chen X, Jia K, Marikar FM and Hua Z: Modification of cyclic NGR tumor neovasculature-homing motif sequence to human plasminogen kringle 5 improves inhibition of tumor growth. PLoS One 7: e37132, 2012.

21. Yang JL, Qin Y, Li L, Cao CY, Wang Q, Li Q, Lv YF and Wang Y: Apoptotic melanoma B16-F1 cells induced by lidamycin could initiate the antitumor immune response in $\mathrm{BABL} / \mathrm{c}$ mice. Oncol Res 23: 79-86, 2016.

22. Tang X, Dai H, Zhu Y, Tian Y, Zhang R, Mei R and Li D: Maytansine-loaded star-shaped folate-core PLA-TPGS nanoparticles enhancing anticancer activity. Am J Transl Res 6: 528-537, 2014.

23. Burns KE, Robinson MK and Thévenin D: Inhibition of cancer cell proliferation and breast tumor targeting of pHLIP-monomethyl auristatin E conjugates. Mol Pharm 12: 1250-1258, 2015 . 